\title{
Perfil Lipídico y sus características entre la Población Infantil
}

\author{
Emilio González Jiménez ${ }^{a}$
}

a Departamento de Enfermería, Facultad de Ciencias de la Salud, Universidad de Granada.

Correspondencia:

Dr. Emilio González Jiménez,

Departamento de Enfermería,

Facultad de Ciencias de la

Salud,

Avda. Madrid s/n, 18071 -

Granada, España.

E-mail: emigoji@ugr.es

Recibido el 1 de mayo de 2011.

Aceptado para su publicación el 18 de junio de 2011.

\begin{abstract}
RESUMEN
En la actualidad se acepta que la aterosclerosis tiene su inicio a edades cada vez más tempranas. En este sentido, profundizar en el estudio del perfil lipídico en el niño constituye una prioridad para la salud pública, ello debido fundamentalmente a su relación con una mayor incidencia de enfermedad cardiovascular en la etapa adulta. Los valores plasmáticos de colesterol total y colesterol ligado a lipoproteínas de baja densidad son muy elevados en la actual población infantil. Ello parece tener su explicación en una dieta poco saludable, hipercalórica y rica en grasas saturadas, si bien estudios recientes indican la existencia de otros factores implicados, como son la genética y ciertas alteraciones hormonales. El objetivo de este trabajo ha sido ofrecer una visión sobre las características del perfil lipídico de la población infantil actual.

Palabras clave. Valores Lipídicos, Niños, Dieta, Genes.
\end{abstract}

\section{ABSTRACT}

Lipid profile characteristics in children

It is currently accepted that atherosclerosis starts at increasingly earlier ages. A more profound study of the lipid profile in children is a priority for public health mainly because of its relationship with a higher incidence of cardiovascular disease in adulthood. Plasma levels of total cholesterol and low density lipoprotein cholesterol are very high in today's children. This seems to be explained by an unhealthy diet that is high in calories and rich in saturated fats. Although recent studies indicate that other factors, such as genetics and certain hormonal disorders, are also involved. The aim of this study was to provide a realistic view of the lipid profile characteristics of children today.

Key words. Lipid Values, Children, Diet, Genes.

\section{INTRODUCCIÓN}

En la actualidad se sabe cómo la aterosclerosis tiene su inicio a edades tempranas de la vida ${ }^{1,2}$. Estudios recientes han demostrado mediante el uso de ultrasonido intravascular que las lesiones coronarias aparecen precozmente en uno de cada seis adolescentes ${ }^{3}$.

Los datos existentes permiten plantear una influencia clara de los factores ambientales en la génesis de la enfermedad cardiovascular a edades tempranas ${ }^{4}$. No obstante, los últimos resultados sugieren la posibilidad de que la enfermedad aterosclerótica quede configurada ya desde la infancia para su posterior desarrollo en la edad adulta. Según esto, Barker y cols. (1997) sugirieron que la cardiopatía isquémica en la edad adulta resultaría de la previa acción de los factores medioambientales en la vida intraútero y en la infancia temprana ${ }^{5}$.

Por otra parte, existe evidencia científica sobre una relación entre las concentraciones lipídicas en niños y adolescentes y las que presentan posteriormente en la edad adulta 6 . Esta disposición de los sujetos a permanecer en el mismo percentil, según edad y sexo, a lo largo del tiempo se conoce como persistencia o tracking. Según esto, toda actividad orientada a controlar 
los valores lipídicos en niños y adolescentes tendrá repercusiones en el desarrollo de trastornos cardiovasculares durante la etapa adulta ${ }^{6}$.

\section{PERFIL LIPÍDICO EN LA INFANCIA. SITUACIÓN ACTUAL}

Uno de los principales estudios orientados en nuestro país al análisis de los factores de riesgo cardiovascular en niños ha sido el estudio Cuatro Provincias $^{7}$. Dicho estudio se llevó a cabo en una población de niños prepuberales pertenecientes a cuatro provincias, analizando sus valores plasmáticos lipídicos. Los resultados obtenidos permitieron concluir que un elevado porcentaje de niños de estas provincias superaba el actual límite de $200 \mathrm{mg} / \mathrm{dl}$ de colesterol. Estos resultados son preocupantes si tenemos en cuenta que las recomendaciones actuales sugieren no exceder los $200 \mathrm{mg} / \mathrm{dl}$ de colesterol total para los adultos. Al respecto, un $25 \%$ de la población escolar sobrepasa dichos valores ${ }^{8}$.

Resultados procedentes de otros estudios muestran cómo hasta un $40 \%$ de los niños con cifras elevadas de colesterol disminuirán los niveles del mismo con la llegada de la madurez sexual ${ }^{9}$. Ahora bien, a pesar de que una parte de estos niños puede normalizar sus valores de lípidos con la pubertad, actualmente se acepta que las lesiones ateroscleróticas comienzan a formarse ya en la más temprana infancia?.

Por otra parte, y de forma paralela a los valores elevados de colesterol total, encontramos cifras de colesterol unido a lipoproteínas de alta densidad (HDL-colesterol) igualmente elevadas, siendo éstas en torno a los $59 \mathrm{mg} / \mathrm{dl}$ de media. Estas cifras de cHDL resultan más elevadas que las encontradas entre la población infantil y juvenil de países como los Estados Unidos según datos del estudio NHANES $1 I^{10}$, en el cuál fueron descritos valores de HDL-colesterol de $52 \mathrm{mg} / \mathrm{dl}$ entre niños de 6 a 8 años.

Si bien es cierto, en relación al HDL-colesterol, que durante las edades valoradas en estos estudios (6 8 años) sus valores no poseen un valor predictivo en tanto que inmediatamente después de la pubarquia, los valores en niñas de HDL-colesterol se elevan, a diferencia de los niños en los que disminuyen ${ }^{11}$. En el caso de la mujer, esta elevación persistirá hasta la menopausia, momento en el cual volverán a equilibrarse ${ }^{11}$.

Con respecto a los valores de colesterol ligado a lipoproteínas de baja densidad (LDL-colesterol), los estudios realizados en nuestro país con población infantil de entre 8 y 9 años de edad ${ }^{12}$ muestran una clara tendencia al alza en sus valores. Esta circunstancia parece tener su explicación en una situación de deterioro progresivo de la dieta con un consumo cada vez mayor de grasas saturadas.

\section{FACTORES CONDICIONANTES DEL PERFIL LIPÍDICO EN POBLACIÓN INFANTIL}

\section{Factores dietéticos}

En España, al igual que en todos los países desarrollados, se ha producido un alejamiento de la alimentación tradicional para progresivamente ir adoptando hábitos y estilos más propios de la cultura anglosajona y americana. Según esto, en la actualidad asistimos a un abandono de la dieta mediterránea pasando a un modelo de alimentación fundamentado en una ingesta desmesurada de proteínas de alto valor biológico, grasas saturadas y carbohidratos de asimilación rápida o simples ${ }^{13}$.

En el estudio desarrollado por González $(2010)^{14}$, a partir de una población de 977 niños y adolescentes escolares de la ciudad de Granada y provincia, se encontró una alimentación hipercalórica, con un evidente exceso de grasa e hidratos de carbono entre la población escolar valorada. Esta situación resultó paralela a una prevalencia de obesidad del $4,98 \%$ en chicos y del $12,70 \%$ entre las chicas. Esta mayor prevalencia de sobrepeso y obesidad, junto con valores elevados de glucosa y triglicéridos, sugiere que estas alteraciones pueden contribuir a un incremento de la mortalidad cardiovascular en la edad adulta.

En España en la actualidad el consumo de aceite de oliva supone la mayor fuente de grasas saludables, fundamentalmente por su contenido en polifenoles y efectos beneficiosos de los mismos 
(propiedades antiinflamatorias y antitrombóticas) sobre el aparato cardio-circulatorio ${ }^{15}$. Así, se sabe cómo los ácidos grasos monoinsaturados tienen una influencia positiva sobre el perfil lipídico infantil y juvenil, disminuyendo el colesterol total y el cLDL, y manteniendo o elevando el $\mathrm{CHDL}^{15}$.

Por su parte, la ingesta de ácidos grasos saturados (AGS) induce un incremento de los valores plasmáticos del CLDL y se asocia con un mayor riesgo cardiovascular en la población adulta. Estudios recientes sugieren cómo la sustitución de este tipo de grasa por ácidos grasos insaturados resulta más eficaz como medida de prevención cardiovascular que la reducción de la ingesta total de grasa ${ }^{15}$. Según esto, la ingesta de ácidos grasos saturados debe ser inferior al $10 \%$ de la ingesta calórica total ${ }^{16}$.

La evidencia disponible muestra cómo la relación entre ácidos grasos saturados y el colesterol plasmático en niños y adolescentes resulta similar a la observada en adultos ${ }^{17}$. El análisis del patrón dietético de la población estudiada por González $(2010)^{14}$ mediante un cuestionario de frecuencia de consumo alimentario puso de manifiesto que la ingesta energética, de grasas y proteínas procedentes del consumo de grasas saturadas fue considerablemente elevada.

El $57 \%$ de las calorías que consumen los escolares de Granada proceden de las grasas, con un $20 \%$ de monoinsaturadas, un $10 \%$ de poliinsaturadas y un $27 \%$ de saturadas ${ }^{14}$. Esta situación posibilita plantear que muchos de los valores elevados de colesterol LDL resultan de la gran cantidad de grasas saturadas que nuestros menores ingieren, y que enmascara el efecto beneficioso de los ácidos grasos monoinsaturados.

En el estudio realizado por Musso y cols. $(2011)^{18}$ en una población de escolares, en el que se analizaba la dieta, se comprobó que la grasa total representaba el $41 \%$ de las calorías totales consumidas. Por su parte, en el estudio Cuatro Provincias la ingesta de grasa total en los niños de Madrid representaba el $47,3 \%$ de las calorías ${ }^{7}$. Este aumento del $6,3 \%$ en el consumo de grasa podría explicar el incremento importante del cLDL.
Un estudio poblacional realizado en Estados Unidos estimó que el riesgo de padecer enfermedad cardiovascular es un $93 \%$ más alto cuando los ácidos grasos saturados reemplazan tan sólo al $2 \%$ de las calorías provenientes de los hidratos de carbono ${ }^{19}$. Los datos del estudio longitudinal de Frammingham (Estados Unidos) mostraron, asimismo, que el riesgo de padecer enfermedad cardiovascular aterosclerótica era significativamente mayor en aquellas personas que consumían margarina en lugar de aceite de oliva ${ }^{20}$. Otros estudios recientes, como el desarrollado por Dalainas y cols. (2008) ${ }^{21}$, pusieron de manifiesto un mayor riesgo de padecer enfermedad cardiovascular aterosclerótica a edades tempranas al ingerir niveles elevados de ácidos grasos saturados.

\section{Factores genéticos}

Los factores genéticos, asimismo, pueden ser determinantes de los valores de lípidos. Entre los polimorfismos de las apolipoproteínas, las alteraciones genéticas de la apo $\mathrm{E}$ han adquirido un papel de singular importancia en el problema de la aterosclerosis ${ }^{22}$.

En numerosos estudios se ha observado la influencia del genotipo de Apo E sobre los valores plasmáticos de lípidos ${ }^{23}$. El estudio de la influencia del genotipo de apo $\mathrm{E}$ sobre los valores de lípidos en la población infantil claramente prepuberal (6 a 8 años) ha demostrado que, en este grupo de edad, el genotipo de apo $\mathrm{E}$ afecta de una manera importante a los valores plasmáticos de colesterol total, cLDL y apo B45 ${ }^{23}$.

Por el contrario, no se han descrito efectos significativos del genotipo de apo E sobre los valores plasmáticos de triglicéridos en niños ${ }^{24}$. Dicha relación sólo se ha observado de forma estrecha en población adulta ${ }^{24}$. En este sentido, los resultados respecto a la influencia de este genotipo sobre valores plasmáticos de triglicéridos en población pediátrica resultan poco concluyentes ${ }^{24}$. Asimismo, se han verificado diferencias en el efecto que el polimorfismo de apo $\mathrm{E}$ ejerce sobre los valores de lípidos y lipoproteínas en adultos de distinto sexo ${ }^{25}$. Esto parece indicar que factores como el sexo y el estado hormonal muestran una implicación respecto 
del diferente efecto del genotipo de apo E sobre los valores de lípidos y lipoproteínas existentes en hombres y mujeres ${ }^{25}$.

Del mismo modo, estudios como el desarrollado por Lumey y cols. $(2009)^{26}$ relacionan el bajo peso al nacer con las concentraciones lipídicas en la edad adulta, encontrado un efecto importante del peso al nacer sobre la influencia que ejerce el genotipo de apo $E$ a nivel de los valores de lípidos. Esta influencia del polimorfismo de apo $\mathrm{E}$ sobre los valores de colesterol total y cLDL parece cobrar mayor fuerza entre niños de peso bajo al nacimiento.

\section{Aspectos dietéticos relacionados con el control de los valores lipídicos en niños}

El control de los niveles de lípidos en la sangre en población infantil pasa necesariamente por el establecimiento de un estilo de vida saludable, es decir, mediante la práctica de ejercicio físico a diario, así como también controlando su alimentación ${ }^{27}$, si bien es cierto que para controlar los valores de lípidos durante la infancia nunca deben establecerse restricciones de grasa y colesterol entre niños menores de 2 años. En este aspecto, la Academia Americana de Pediatría establece que los niños de entre 2 y 18 años deben mantener una ingesta de ácidos grasos saturados y de grasa total por debajo del $20 \%$ de la ingesta calórica total ${ }^{28}$.

Por tanto, el perfil nutricional de escolares en edad prepuberal puede ser corregido mediante intervenciones nutricionales orientadas a vigilar la ingesta calórica y a limitar el consumo de grasas saturadas y colesterol ${ }^{14}$. En este sentido, y como estrategia efectiva, cabría plantear la necesidad de reducir la ingesta de alimentos ricos en colesterol y grasas saturadas como la bollería industrial, entre otros muchos, por otros alimentos de menor contenido en grasas $^{14}$.

En modo paralelo, y con objeto de mantener una alimentación saludable, debería fomentarse el consumo de alimentos ricos en hidratos de carbono complejos, presenten en alimentos como el pan, la pasta o el arroz $^{14}$. Sólo en aquellos casos de niños con valores lipídicos muy elevados y persistentes, descendientes de padres portadores de dislipemias genéticas (hipercolesterolemia familiar heterocigota e hiperlipoproteinemia familiar combinada) asociadas a obesidad o diabetes podrá ser necesario recurrir a tratamientos farmacológicos ${ }^{29}$.

Considerando todo lo expuesto, parece quedar claro que los valores lipídicos en los niños son resultado de una profunda interacción entre factores genéticos, nutricionales y otros aspectos más recientemente descritos como el peso al nacer o su situación hormonal.

\section{BIBLIOGRAFÍA}

1. Semeran K, Bossowski A. Changes in the cardiovascular system in selected endocrinopathies in children. Pediatr Endocrinol Diabetes Metab. 2011; 17(1):42-7.

2. Kolovou GD, Bilianou $\mathrm{H}$, Mikhailidis DP. Postprandial lipemia in children and adolescents. Curr Vasc Pharmacol. 2011; 9(3):318-20.

3. Mackay MT, Wiznitzer M, Benedict SL, Lee KJ, Deveber GA, Ganesan V. Arterial ischemic stroke risk factors: the International Pediatric Stroke Study. Ann Neurol. 2011; 69(1):130-40.

4. Gilardini L, Pasqualinotto L, Di Matteo S, Caffetto K, Croci M, Girola A, Invitti C. Factors Associated With Early Atherosclerosis and Arterial Calcifications in Young Subjects With a Benign Phenotype of Obesity. Obesity. 2011; 27[Epub ahead of print].

5. Beauloye V, Zech F, Tran HT, Clapuyt P, Maes M, Brichard SM. Determinants of early atherosclerosis in obese children and adolescents. J Clin Endocrinol Metab. 2007; 92(8):3025-32.

6. Liu YL, Liang HR, Liu HT, Li SY, Zhou YY, Cheng HL, Zhou LS. Association of serum adiponectin levels with artherosclerosis and the metabolic syndrome in obese children. J Pediatr Endocrinol Metab. 2010; 23(8):743-51.

7. Garcés C, Lasunción MA, Ortega H, López Cubero L, Benavente M, Rubio I, et al. Factores metabólicos en población escolar asociados a la mortalidad cardiovascular en los adultos: Estudio Cuatro Provincias. Med Clin (Barc). 2002; 118:767-70.

8. Yalçin SS, Günes $B$, Unal $S$, Gümrük $F$, Coçkun $T$. Antihyperlipidemic agents cause a decrease in von Willebrand factor levels in pediatric patients with familial hyperlipidemia. J Pediatr Endocrinol Metab. 2010; 23(8):765-71.

9. Tan F, Okamoto M, Suyuma A, Miyamoto T. Tracking of cardiovascular risk factors and a cohort study on hyperlipidemia in rural schoolchildren in Japan. J Epidemiol. 2000; 10:255-61.

10. Hickman TB, Briefel RR, Carroll MD, Rifkind BM, Cleeman $\mathrm{JI}$, Maurer KR, et al. Distribution and trends of serum lipid levels among United States children and adolescents ages 4-19 years: data from the Third National Health and Nutrition Examination Survey. Prev Med. 1998; 27:879-90.

11. Bestehorn K, Jünger C, Smolka W, Gitt AK. Regional differences in the treatment of dyslipidemia in Germany. Dtsch Med Wochenschr. 2011; 136(11):512-8.

12. Plana T, Gracia R, Méndez I, Pintor L, Lazaro, CastroFornieles J. Total serum cholesterol levels and suicide attempts in child and adolescent psychiatric impatients. Eur Child Adolesc Psychiatry. 2010; 19(7):615-9.

13. Rodríguez Artalejo F, Banegas JR, Graciani A, HernándezVecino R, del Rey-Calero J. Food supply versus household survey data: nutrients consumption trends for Spain, 19581988. Eur J Epidemiol. 1996;12:367-71. 
14. González Jiménez E. Evaluación de una intervención educativa sobre nutrición y actividad física en niños y adolescentes escolares con sobrepeso y obesidad de Granada y provincia. Tesis Doctoral. Universidad de Granada, 2010.

15. Aguilar Cordero MJ, González Jiménez E, Sánchez Perona J, Padilla López CA, Álvarez Ferre J, Mür Villar N et al. The Guadix study of the effects of a Mediterranean diet breakfast on the postprandial lípido parameters of overweight ando bese pre-adolescents. Nutr Hosp. 2010; 25(6):1025-33.

16. Frankel EN. Nutritional and biological properties of extra virgin olive oil. J Agric Food Chem. 2011; 59(3):785-92.

17. Pérez $P$, García $A$, Delgado J, Pérez F, López J. Mediterranean Diet Rich in Olive oil and Obesity, Metabolic Syndrome and Diabetes Mellitus. Curr Pham Des. 2011; 17(8):769-77.

18. Musso C, Graffigna M, Soutelo J, Honfi M, Ledesma L, Miksztowicz V et al. Cardiometabolic risk factors as apolipoproteína $\mathrm{B}$, triglyceride/HDL-colesterol ratio and C-reactive proteína, in adolescents with and without obesity: cross-sectional study in middle class suburban children. Pediatr Diabetes. 2011; 12(2):229-34.

19. Castro-Beiras A, Muñiz J, Juane R, Suárez Barros J, Santamaría García JL, Velasco Horta B, et al. Estudio Brigantium. Factores de riesgo cardiovascular en la niñez y adolescencia en un área rural gallega. Med Clin (Barc). 1993; 100:481-7.

20. Elcarte R, Villa I, Sada J, Gascó M, Oyarzabal M, Solá A. Estudio de Navarra (PECNA). Hiperlipemias II. Variaciones de los niveles medios de colesterol total, colesterol LDL y triglicéridos de una población infanto-juvenil según edad y sexo. An Esp Pediatr. 1993; 38:159-66.
21. Dalainas I, loannou HP. The role of trans fatty acids in atherosclerosis, acrdiovascular disease and infant development. Int Angiol. 2008; 27(2):146-56.

22. Bazzaz JT, Nazari M, Nazem H, Amiri P, Fakhrzadeh H, Heshmat $R$ et al. Apolipoprotein $E$ gene polymorphism and total serum cholesterol level in Iranian population. J Postgrad Med. 2010; 56(3):173-5.

23. Landázuri $\mathrm{P}$, Loango N, Gallego ML, Restrepo B. Gender, age and plasma lipids differences associated with apolipoproteína $E$ polymorphism in school children. Biomedica. 2009; 29(3):382-91.

24. Smart MC, Dedoussis G, Louizou E, Yannakoulia M, Drenos F, Papoutsakis C et al. APO E, CETP and LPL genes show strong associaction with lipid levels in Greek children. Nutr Metab Cardiovasc Dis. 2010; 20(1):26-33.

25. Lunegova OS, Pavlenko MA, Kerimkulova AS, Noruzbaeva AM, Samanchina BT, Zalesskaia luV et al. Association of the serum level of apolipoprotein $\mathrm{E}$ with coronary artery disease and carotid artery atherorclerosis in Kyrgyz men with dyslipidemia. Ter Arkh. 2010; 82(9):18-23.

26. Lumey LH, Stein AD, Kahn HS, Romijn JA. Lipid profiles in middle-aged men and women after famine exposure during gestation: the Dutch Hunger Winter Families Study. Am J Clin Nutr. 2009; 89(6):1737-43.

27. Ostojic SM, Stojanovic M, Stojanovic V, Maric J. Adiposity, physical activity and blood lipid profile in 13 year ols adolescents. J Pediatr Endocrinol Metab. 2010; 23(4):33343.

28. American Academy of Pediatrics. Cholesterol in childhood. Pediatrics. 1998; 101:141-7.

29. Pozzato C, Verduci E, Scaglioni S, Radaelli G, Salvioni M, Rovere A et al. Liver fat change in obese children alter a 1 year nutrition behavior intervention. J Pediatr Gastroenterol Nutr. 2010; 51(3):331-5. 